\title{
Research on Channel Modeling and Communication Coverage of Wireless Sensor Networks in Barrier Area of Nuclear Power Plants
}

\author{
Zhi-Guang Deng $\mathbb{D}^{\mathbb{D},}$ Qian Wu, Xin Lv, Bi-Wei Zhu, Mei-Qiong Xiang, Xue-Mei Wang, \\ and Jia-Liang Zhu
} Science and Technology on Reactor System Design Technology Laboratory, Nuclear Power Institute of China,
Chengdu 610213, China

Correspondence should be addressed to Zhi-Guang Deng; dzg7400613@163.com

Received 17 August 2021; Revised 20 January 2022; Accepted 9 February 2022; Published 7 March 2022

Academic Editor: Arkady Serikov

\begin{abstract}
Copyright (c) 2022 Zhi-Guang Deng et al. This is an open access article distributed under the Creative Commons Attribution License, which permits unrestricted use, distribution, and reproduction in any medium, provided the original work is properly cited.

In view of the multimetal barrier environment of nuclear power plant, by considering the factors such as transmission power, transmission position, and multipath interference, based on the simulation of metal pipes and equipment, this paper carries out the barrier area channel modeling in logarithmic fading mode and makes quantitative analysis on the channel transmission, path loss, channel power characteristics, and so on under the metal barrier environment. Based on the channel modeling, this paper optimizes the coverage of the network in the obstacle area by using the improved teaching and learning group intelligent algorithm. The simulation results show that the improved teaching and learning algorithm can optimize the network coverage of the obstacle area well, and under the four obstacle modules, 14 nodes can cover the whole area by more than $99 \%$. This provides a solution to the problem of network coverage in the practical application of wireless sensor networks.
\end{abstract}

\section{Introduction}

As a new network emerging in recent years, wireless sensor networks can eliminate signal transmission cables, and some even cancel power supply cables. At present, there are many mature applications in transportation, petrochemical, electric power, water treatment, and other industries, but it is still blank in the field of nuclear power plants. Some particularities of naval nuclear power plants pose challenges to the application of wireless sensor networks, such as the attenuation of wireless signals caused by complex cabin structure [1], the real-time network under high-density sensor arrangement, the electromagnetic compatibility under complex electromagnetic environment, and the safety and reliability of wireless transmission networks [2], but the research in this area is basically blank.

The wireless channel is transmission media for mobile communication, and all information is transmitted in this channel. The channel performance directly determines the quality of people's communication. Therefore, in order to transmit useful information with high quality and large capacity on limited spectrum resources, it is necessary to know the channel characteristics clearly. Then, according to the characteristics of the channel, a series of anti-interference and antifading measures are taken to ensure the requirements of transmission quality and transmission capacity. In order to study the wireless communication technology, it is necessary to have a wireless channel model and channel estimation method which are consistent with the actual transmission environment. Literature [3] studies wireless channel modeling based on 3D ray tracing. Literature [4] simulates the channel transmission path by using SystemView software based on iterative algorithm and studies the modeling of wireless channel transmission in confined space. Some researchers have also discussed and studied machine learning-based channel modeling, and the study found that this approach is a promising modeling method [5-7].

With the development of network communication technology, some communication requirements in extreme 
environments that cannot be covered by traditional networks are emerging day by day, such as underground network communication, field sensor network communication, and deep space network communication. Data communication in this kind of environment usually has the characteristics of large signal delay, high channel bit error rate, low carrier-to-noise ratio/signal-to-noise ratio, frequent interruption of communication links, etc., and the data transmission performance is facing a huge challenge. Influenced by environmental factors, it is necessary to show the research of wireless communication transmission technology based on complex environment.

There are a large number of metal obstructions in the environment of nuclear power plant, which have a great impact on wireless communication. Meanwhile, multipath effect and shadow fading are obvious in wireless communication in this environment. Therefore, this paper focuses on the following:

(1) A modeling study of channel strength for wireless communication in obstructive areas was conducted to ensure that nodes can obtain sufficient received power as well as communication correctness

(2) An optimal analysis of network coverage in obstructive areas was also carried out by improving the swarm intelligence algorithm for teaching and learning

(3) Finally, based on the simulation results of (2), the actual test was verified in a multimetal barrier bench environment, and it is known that the simulation results of (2) can bring guidance to the actual wireless sensor optimization arrangement

Therefore, this paper studies the channel strength of wireless communication in obstacle areas by modeling to ensure that nodes can obtain enough received power and accuracy, and the network coverage of obstacle areas is optimized.

\section{Modeling and Analysis of Wireless Channel in Narrow Multimetal Environment}

In the cabin of nuclear power plant, there are a large number of metal pipes and equipment, and wireless communication in such an environment will be affected by the surrounding environment, so multimetal interference environment has become one of the typical scenarios of wireless channel research. In order to ensure the accuracy and efficiency of wireless channel transmission information, it is necessary to model and analyze the wireless channel in this environment. Usually, the metal equipment is steel alloy, and the wireless channel interacts with the irregular metal surface many times in this environment. After reflection, diffraction, and so on, it will produce complex channel propagation path. According to the above problems, the wireless channel model is established for theoretical analysis.

First, the environment is modeled and the specific characteristics such as the location and shape of metal equipment inside are analyzed. Then, the deterministic modeling is carried out according to the analyzed environmental characteristics. Finally, the loss modeling of multipath propagation in wireless channel is obtained according to the equation derivation.

2.1. Environmental Model Building. The built environment model is set as an enclosed cabin for storing nuclear power instruments and equipment, and the theoretical diagram of random sheltered environment is shown in Figure 1. All the research will be based on the sheltered environment.

2.2. Wireless Channel Modeling. According to the known environmental information, this paper models the cabin with an area of about 100 square meters as the research object and the metal material is steel alloy by default. Assuming that the room temperature is $20^{\circ} \mathrm{C}$, the relative dielectric constant of steel alloy is 14.2 and the electrical conductivity is $9.93 * 106(\mathrm{~S} / \mathrm{m})$. The signal transmitting source is unit step pulse, and the transmitting power is $10 \mathrm{~W}$. The cubic steel alloy metal block is established by using MATLAB software. The signal transmitting coordinates $[0,0,15]$ are set in the center of the metal block, and the transmitting times are set to 200 times. Finally, the transmitter angle is defined. According to the above information, wireless channel modeling is completed.

Because of the interference of metal obstacles, the wireless channel will inevitably produce multipath propagation such as direct propagation, reflection, and refraction, and the existence of multipath propagation will result in the path loss of the wireless channel. According to the logarithmic fading equation of large-scale fading, the path loss model of wireless channel is established by calculating and deducing the equation.

Path loss of channel propagation in a limited area is similar to the logarithmic distance model [8]:

$$
\operatorname{PL}(d)=\operatorname{PL}\left(H-H_{c}\right)+10 n \lg \left[\frac{d}{\left(H-H_{c}\right)}\right]+X_{\theta},
$$

where $n$ represents the path loss index, which indicates that the path loss increases with the increase of the transmitting antenna distance, and depends on the structure, material, and type of the surrounding environment. $\mathrm{PL}\left(\mathrm{H}-\mathrm{H}_{c}\right)$ is the path loss of height difference of $H-H_{c}$, and the standard deviation is a normal random variable, which describes the shadowing effect. Shadowing effect refers to the random changes of path loss values at different locations at the same distance under different environments. Usually, the linear fitting method is used to estimate propagation loss and standard deviation according to test data. The logarithmic distance model is the most basic path loss model, which can predict the path loss value of multiple locations.

Generally speaking, the path loss of transmitting and receiving antennas is a linear function of logarithm. By simulating the attenuation caused by antenna height change, a related factor, namely, High Loss Factor (HLF), can be introduced into the traditional log-distance path loss model. In addition, the height of the antenna significantly affects the 


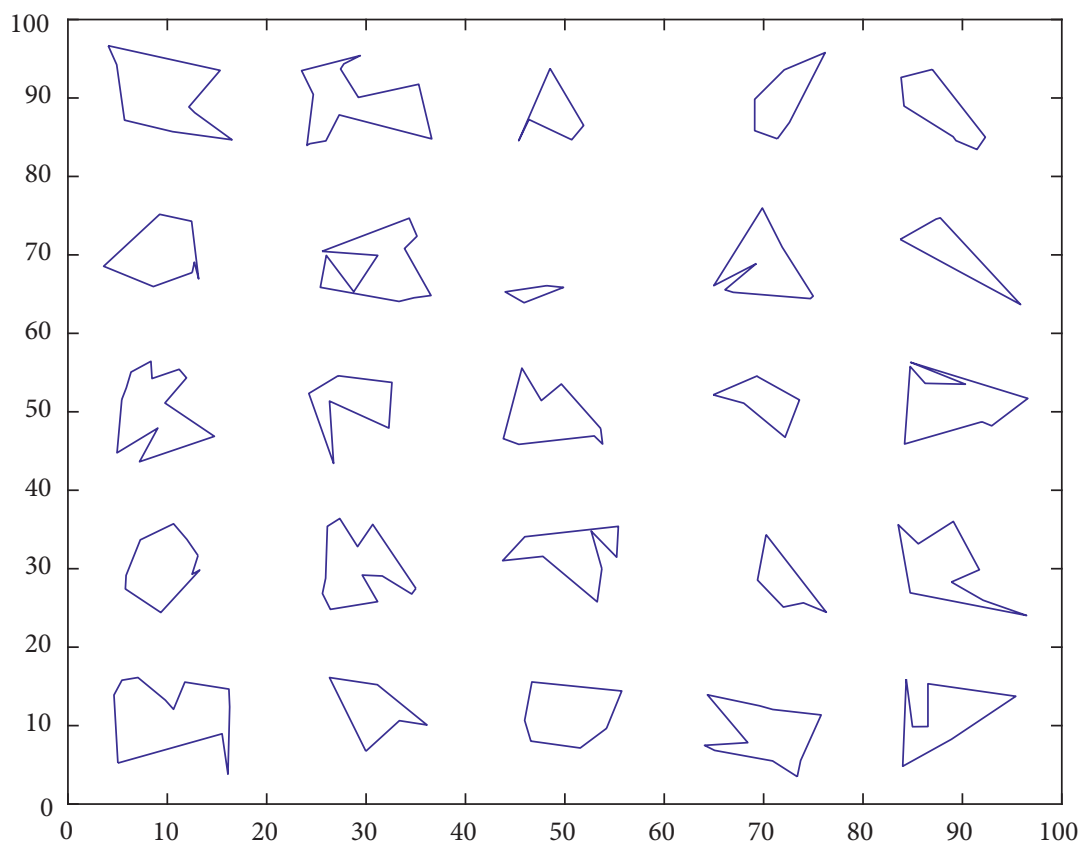

Figure 1: Schematic diagram of complex occlusion environment.

shadow. Therefore, the highly correlated path loss model can be expressed as

$$
\operatorname{PL}(d)=\operatorname{PL}\left(d_{0}\right)+10 n \lg \left(\frac{d}{d_{0}}\right)+\operatorname{HLF}(h)+X(h),
$$

where $\operatorname{PL}\left(d_{0}\right)$ is the path loss at the reference point; $n$ is defined as the path loss index; $\operatorname{HLF}(h)$ is a height attenuation factor, which depends on the height of the transmitting antenna; $X(h)$ is a mean Gaussian random variable, and its standard error depends on the height of receiving antenna, which characterizes the shadowing effect of path loss. The expression of HLF is

$$
\operatorname{HLF}(h)=D_{0}\left[\lg \left(\frac{h}{E_{0}}\right)\right]^{2}+F_{0} .
$$

$D_{0}, E_{0}$, and $F_{0}$ should be extracted from limited scenes. The path loss measured by the transmitting and receiving antenna at distance $D_{0}$ can be calculated by the following expression:

$$
\operatorname{PL}(d)=-10 \lg \frac{1}{m n s} \sum_{i=1}^{m} \sum_{j=1}^{n} \sum_{k=1}^{s}\left|H\left(f_{i}, t_{j}, p_{k}, d\right)\right|^{2},
$$

where $H\left(f_{i}, t_{j}, p_{k}, d\right)$ represents the measured complex frequency response, $f_{i}(i=1, \ldots, m)$ represents observed $m$ frequency points, $t_{j}$ represents $n$ discrete time points, and $p_{k}(i=1, \ldots, s)$ represents grid points during measurement. Average path loss of reference point $\operatorname{PL}\left(d_{0}\right)$ can be described by the following path loss equation:

$$
\operatorname{PL}\left(d_{0}\right)=-10 \lg \left[\frac{c^{2}}{16 \pi^{2} d_{0}^{2} f^{2}}\right] \text {. }
$$

In the narrow multimetal barrier environment of nuclear power plant, besides the channel loss, there are serious multipath propagation phenomena, which need to be modeled mathematically. Figure 2 is a schematic diagram of multipath reflection model, in which $S_{0}$ is the emitting point and $D$ is the receiving point.

The wireless channel parameters of ray path are as follows: $\left\{S, a_{s}, \varphi_{s}, \Omega_{T, s}, \Omega_{R, s}\right\}$, where $S$ is the number of scattering paths, $a_{s}$ is the amplitude of paths, $\varphi_{s}$ is the arrival time of paths, $\Omega_{T, s}$ is the departure direction, and $\Omega_{R, s}$ is the arrival ray directions. The wireless channel parameters of the line-of-sight path are $\left\{a_{0}, \varphi_{0}, \Omega_{T, 0}, \Omega_{R, 0}\right\}$. The modified ray response of scattering path is described as a function of delay, departure direction, and arrival direction and as polarization and reflection coefficient [9].

$$
\begin{aligned}
h\left(\phi, \Omega_{T}, \Omega_{R}\right)= & h_{\text {los }}\left(\phi, \Omega_{T}, \Omega_{R}\right)+h_{s}\left(\phi, \Omega_{T}, \Omega_{R}\right), \\
h_{\text {los }}\left(\phi, \Omega_{T}, \Omega_{R}\right)= & a_{0} \delta\left(\phi-\phi_{0}\right) \delta\left(\Omega_{T}-\Omega_{T, 0}\right) \\
& \cdot \delta\left(\Omega_{R}-\Omega_{R, 0}\right), \\
h_{s}\left(\phi, \Omega_{T}, \Omega_{R}\right)= & \sum_{s=1}^{s} a_{s} \delta\left(\phi-\phi_{s}\right) \delta\left(\Omega_{T}-\Omega_{T, S}\right) \\
& \cdot \delta\left(\Omega_{R}-\Omega_{R, S}\right), \\
a_{S}= & \left\{a_{S, U} \cdot \Gamma \cdot p\right\} \text { or }\left\{a_{S, H} \cdot \Gamma \cdot p\right\}, \\
\Omega_{T, S}= & \left\{\Omega_{T, S, U}\right\} \text { or }\left\{\Omega_{T, S, H}\right\}, \\
\Omega_{R, S}= & \left\{\Omega_{T, S, U}\right\} \text { or }\left\{\Omega_{T, S, H}\right\}, \\
\varphi_{S}= & \left\{\varphi_{S, U}\right\} \text { or }\left\{\varphi_{S, H}\right\} .
\end{aligned}
$$




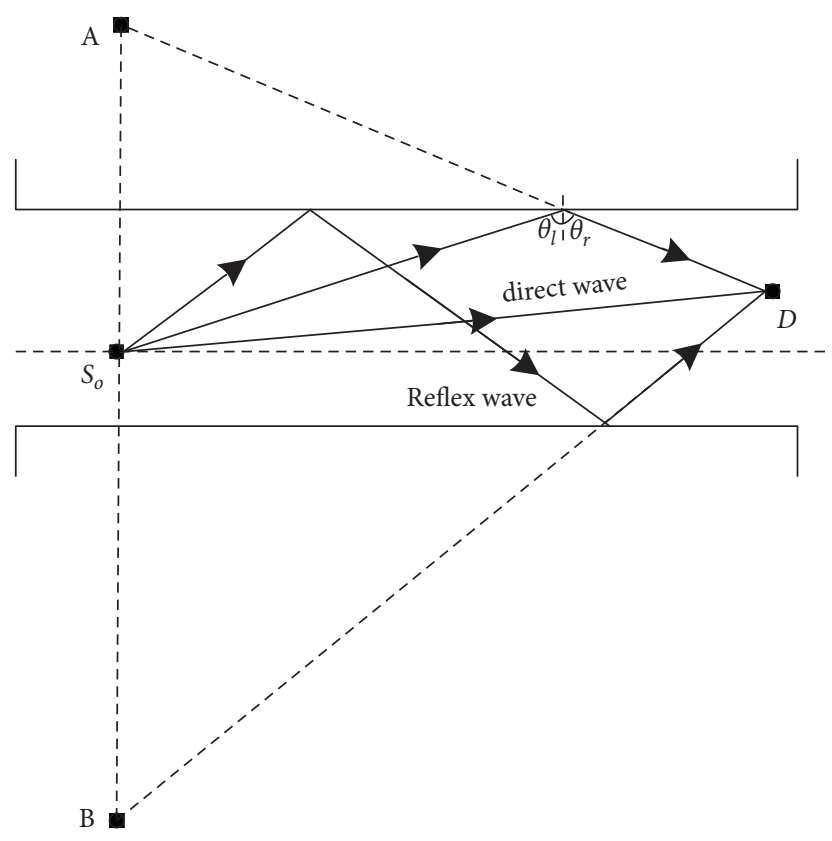

Figure 2: Multipath reflection model.

Equation (7) represents the directional path, and equation (8) represents the scattering line, where $a_{s}$ is the amplitude of scattered rays and $a_{S, V}$ and $a_{S, H}$ are the vertical and horizontal polarization amplitudes of the directional path, respectively. It is assumed that all linearly scattered rays receive the $R_{x}$ antenna within the angular range of $\Omega_{R}$, and $\Omega_{T, s}, \Omega_{R, s}$, and $\varphi_{s}$ represent the vertical and horizontal directions of departure, arrival, and delay, respectively. The power delay distribution characteristics of scattering line based on antenna radiation pattern and polarization are shown in the(10) following equation:

$$
Q\left(\varphi, \Omega_{T}, \Omega_{R}\right)=Z\left\{\left|h\left(\varphi, \Omega_{T}, \Omega_{R}\right)\right|\right\} .
$$

The received signal is composed of many refracted waves and scattered waves with different fading, time delay, and phase shift, and its broadband channel statistical model can be expressed by the classical time-invariant impulse response channel model. The channel has invariance in a symbol period, which can be simplified as

$$
h(t)=\sum_{l=0}^{L} r_{l} \delta\left(t-\tau_{l}\right) e^{j \theta_{l}} .
$$

Assuming that the transmitting power of the transmitter is $p_{t}$ and the receiving power of the receiver is $p_{r}$, the receiving power is shown as follows:

$$
p_{r}=\frac{p_{t} G_{t} G_{r} \lambda^{2}}{4 \pi^{2} d^{2}}
$$

where $G_{t}$ and $G_{r}$ represent the antenna gains of the transmitting antenna and the receiving antenna and $\lambda$ is the wavelength of electromagnetic wave.
2.3. Wireless Channel Propagation Analysis. Two points are arbitrarily selected from the physical space, and their channel propagation paths are shown in Figure 3.

It can be seen from Figure 3 that more than one channel path propagates between any two points, including direct path and reflection path. Because there are few obstacles, only two propagation paths are simulated here. The number of transmissions is set to 200, the channel transmission power is set to $10 \mathrm{~W}$, the transmission point is selected in the center of the metal obstacle to make the channel pass through the obstacle, and the reflection point is shown in Figure 4 .

It can be seen that the wireless channel path will pass through obstacles and the reflection points are not uniform according to the different emission angles and positions, materials, and irregular surfaces of obstacles. It shows that the ability of wireless channel to cross metal obstacles is affected by many aspects, and the denser area is the channel path with strong signal and suitable transmission angle.

After setting the above parameters, the coordinates of the transmitting point are set to $[0,0,15]$ and the transmitting angle is set to calculate the path fading. According to the path fading, different received power intensity is calculated, and the path loss is divided into six different levels by using six colors. The path loss is shown in Figure 5.

In Figure 5, the red line indicates the minimum path loss and the yellow line indicates the maximum path loss. It can be seen from Figure 5 that the closer the channel is to the transmitting point and the less it passes through refraction and scattering, the less the loss is; the farther it is away from the transmitting point and the more times it passes through refraction and scattering, the maximum the path loss is.

According to the above design, the channel communication delay is analyzed, and the input signal is an impulse response function. The Saleh-Valenzuela model ( $\mathrm{S}-\mathrm{V}$ model for short) is established, which is a perfect indoor environment multipath propagation model with clear physical concept and concise mathematical description. Also, the shock response of the $\mathrm{S}-\mathrm{V}$ model is shown in Figure 6.

According to the $\mathrm{S}-\mathrm{V}$ model, the biggest characteristic of multipath propagation is cluster propagation, and the energy and amplitude of multipath obey double exponential distribution and Rayleigh distribution, respectively. Therefore, the $\mathrm{S}-\mathrm{V}$ model is a channel model based on the distribution of multipath signals according to clusters. In this model, multipath components are described according to cluster arrival time (as shown in Figure 7; the solid line is the ideal state, and the dotted line is the simulation result) and path arrival time (as shown in Figure 8), which are two independent Poisson processes: one Poisson process describes the arrival time of the first path of each cluster, and the other Poisson process describes the arrival time of each path in the cluster [10]. The propagation attenuation of cluster and the propagation attenuation of cluster inner diameter are independent of each other. The general expression of impulse response in the $\mathrm{S}-\mathrm{V}$ model [11] is 


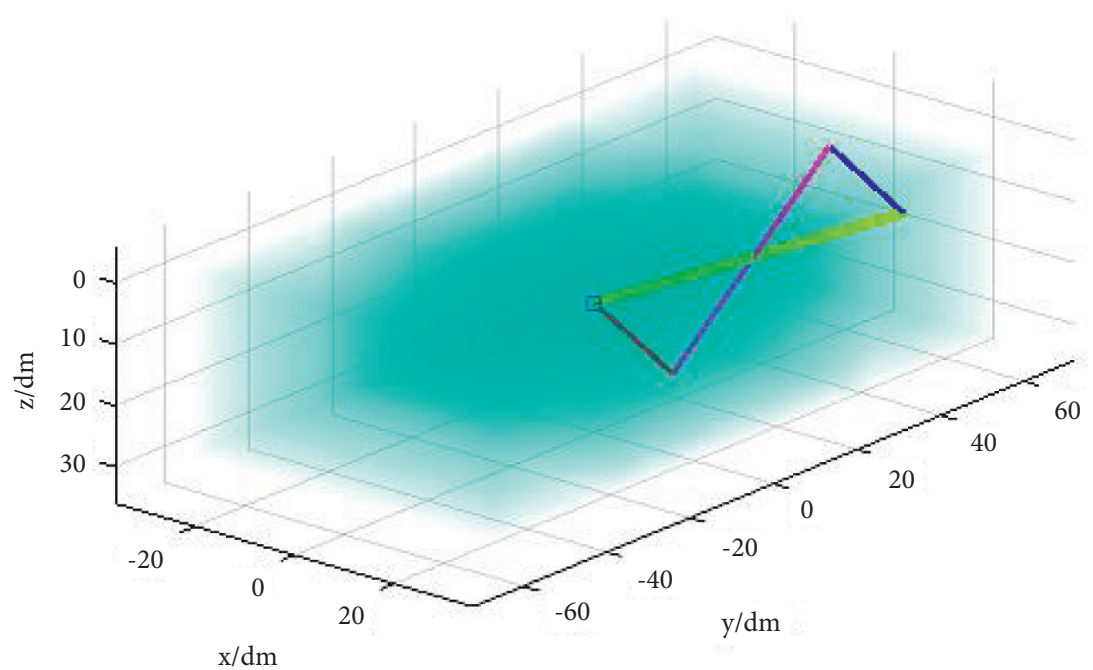

FIgURe 3: Any two-point channel propagation path.

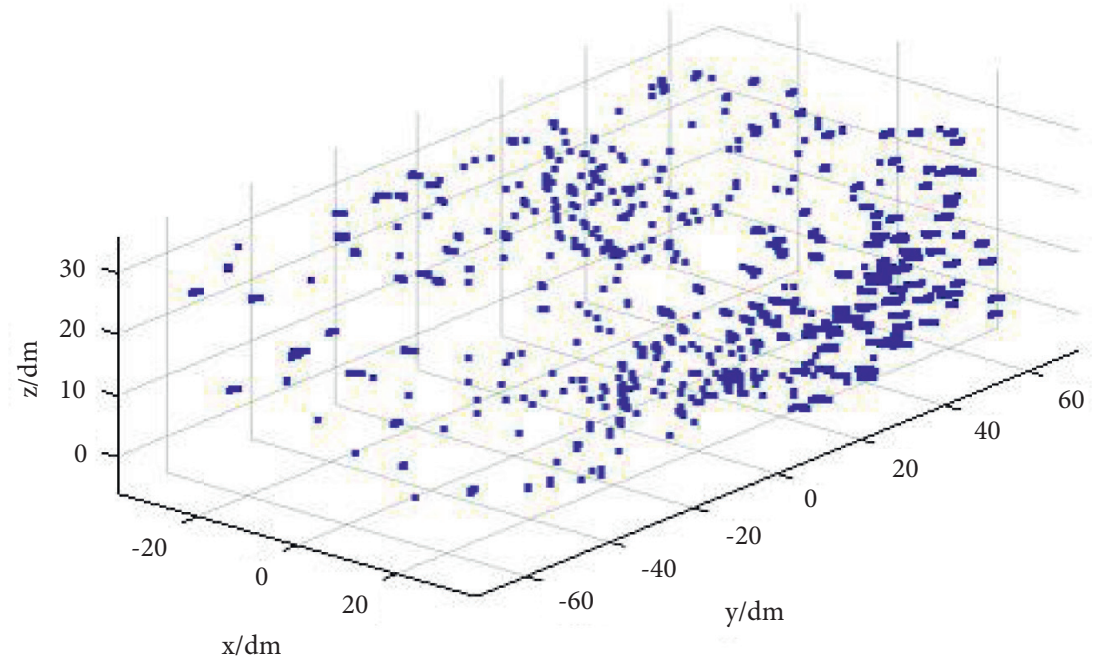

FIgURE 4: Influence of metal on wireless channel.

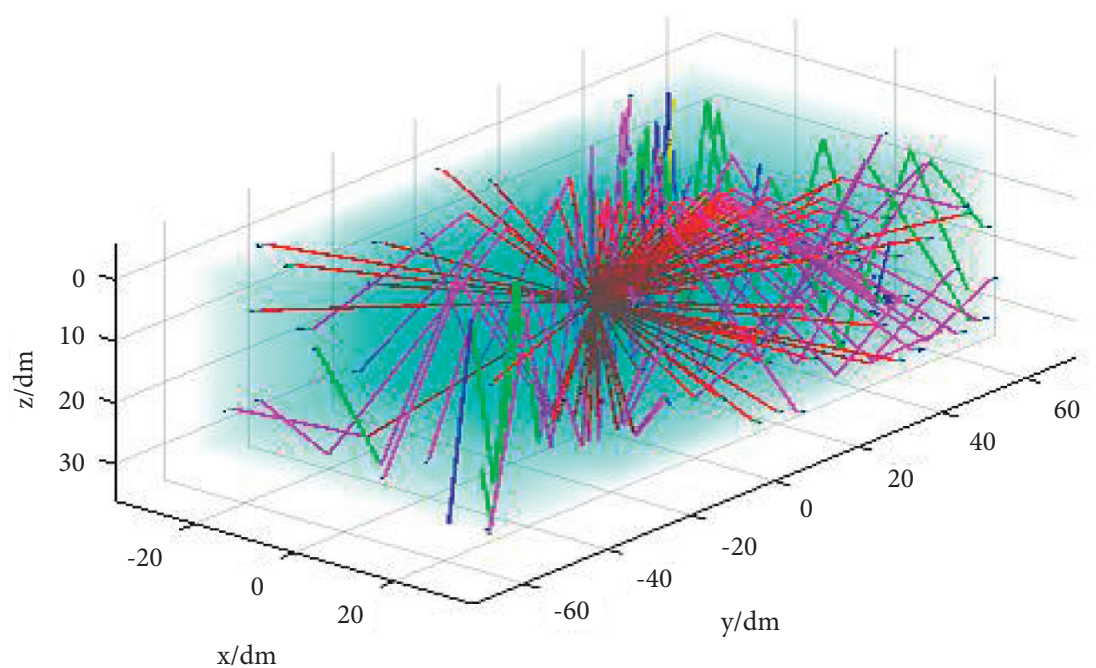

Figure 5: Path loss of any point propagating around. 


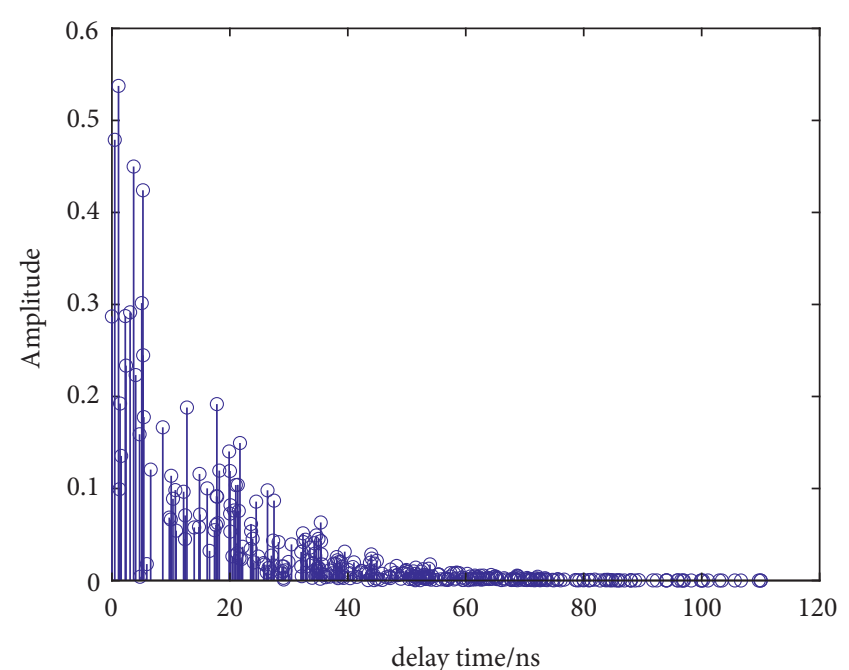

Figure 6: Impact response function of the S-V model.

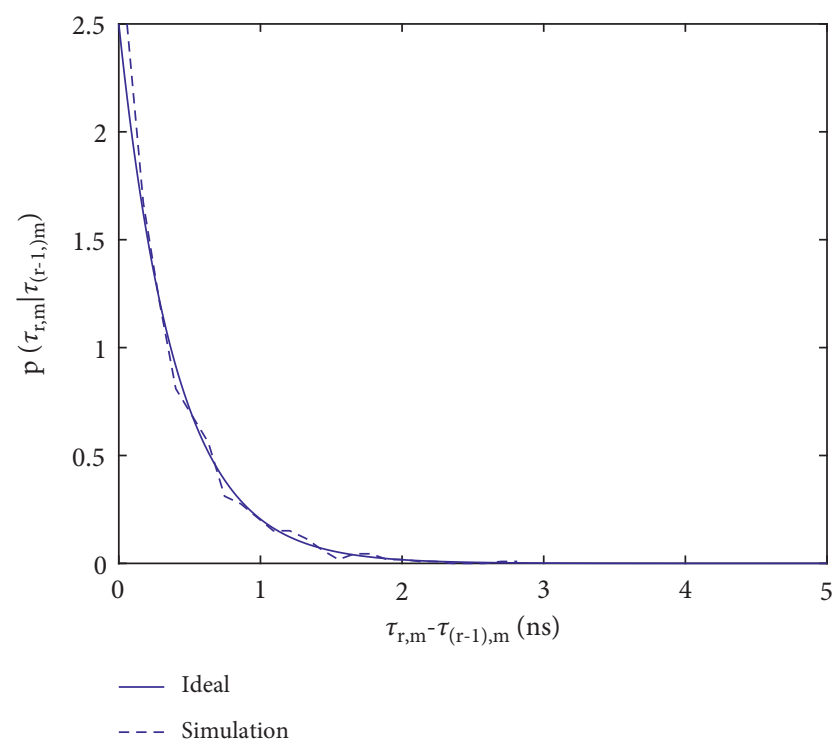

Figure 7: Time distribution of cluster arrival in the S-V model.

$$
h(t)=\sum_{l=0}^{\infty} \sum_{k=0}^{\infty} \beta_{k l} e^{j \theta k l} \delta\left(t-T_{l}-\tau_{k l}\right)
$$

The transmitted signal is regarded as an ideal impulse signal, and the received signal will appear as a pulse. Because of the obstruction of obstacles, the signal received by the receiving end is a multipath composite signal with different paths and time differences, and the composite signal will have time delay spread relative to the original signal in time domain. Delay spread is an important parameter to characterize wireless channel characteristics. The delay equation is as follows [12]:

$$
p(\tau) \approx \overline{|h(t ; \tau)|^{2}}
$$

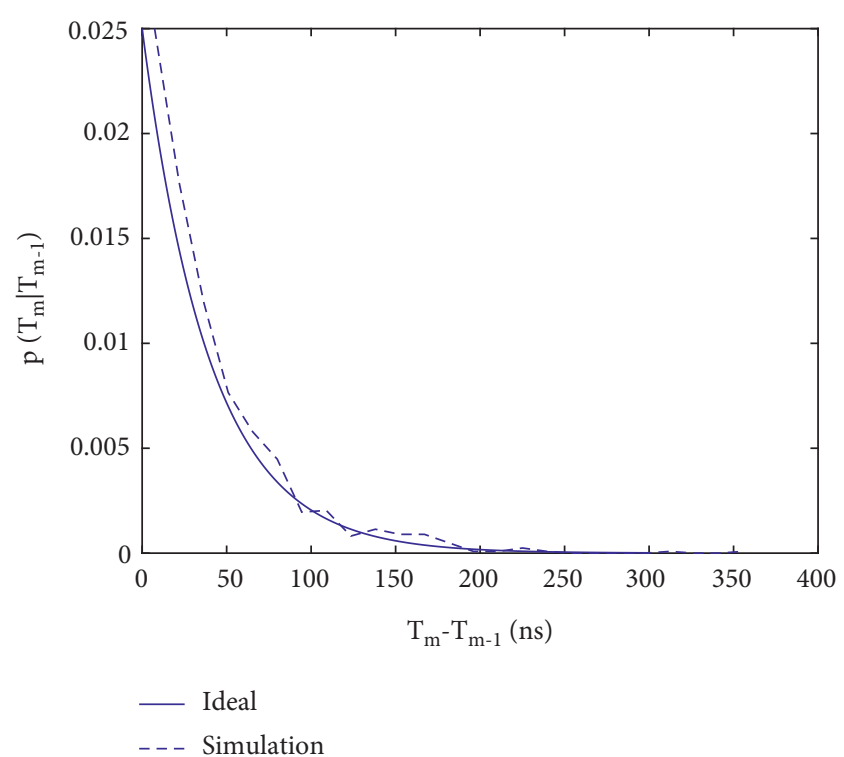

FIgURE 8: Time distribution of path arrival in the S-V model.

To analyze the channel power characteristics, it is necessary to analyze the power delay spectrum, and its equation is as follows:

$$
h^{2}(t)=\sum_{n=1}^{N}\left|a_{n}\right|^{2} \delta\left(t-\tau_{n}\right) .
$$

It can be seen from Figure 9 that the power delay spectrum can be clearly divided into several different groups. The multipath power in each group attenuates exponentially, and the power in the starting path of the next group exceeds the minimum power of the adjacent previous group. Such multipath groups are called "clusters," and the maximum value between each cluster also decreases exponentially. The idealized power delay spectrum is shown in Figure 10. By comparing with Figure 9, it can be seen that the metal environment makes the channel decrease less smooth.

According to the analysis of Figure 4, it can be concluded that the closer to the surface of a smooth object, the less energy is lost when selecting the transmitting position of the wireless channel. It can be seen from Figure 5 that the closer the receiving end of the wireless channel is to the transmitting end, the more accurate and fast the received signal will be. Figures 7 and 8 show that a single metal has little influence on the wireless channel, but the complexity of metal operation and metal actual situation is not considered. It can be seen from Figure 9 that metal has a great influence on the received power cluster, which is caused by multipath transmission affecting the wireless channel.

\section{Analysis of Communication Coverage in Barrier Area of Nuclear Power Plant}

Network communication coverage is the key factor that affects whether the network can communicate or not. The size of network coverage can directly reflect the advantages 


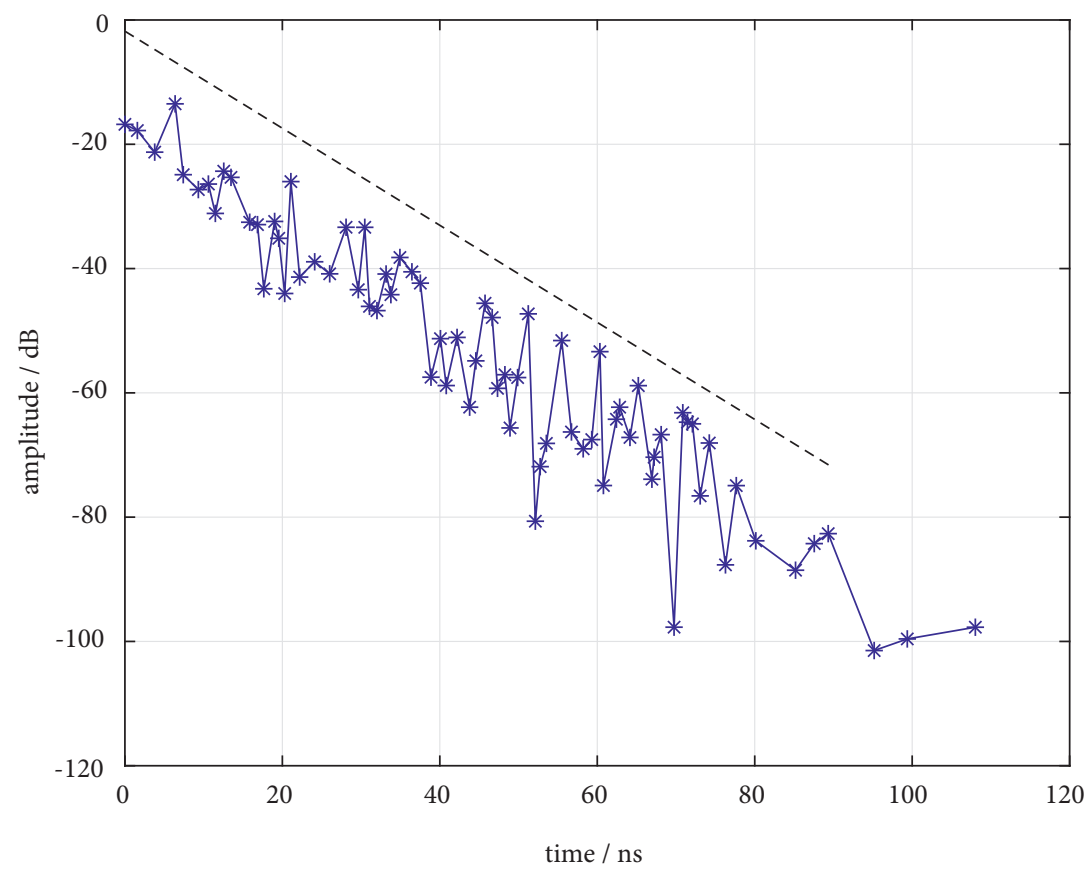

FIGURE 9: Clustering power delay spectrum at different positions.

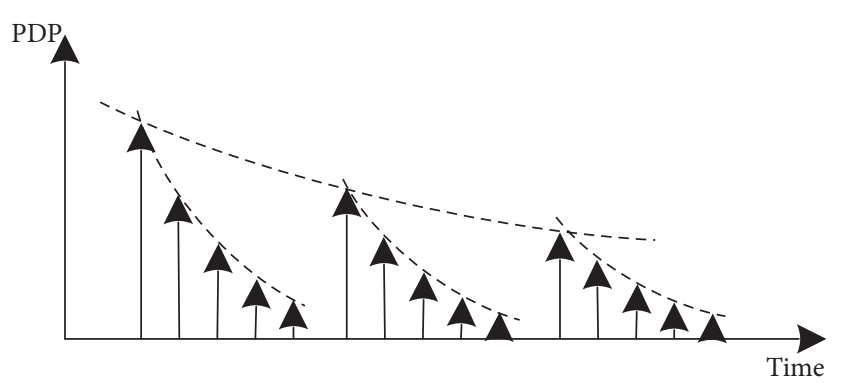

FIgURE 10: Idealized power delay spectrum.

and disadvantages of network coverage. In the deployment process, nodes in wireless sensor networks are prone to node redundancy and coverage blind area, which leads to low network coverage, especially in multimetal barrier areas. How to improve the network coverage is one of the key points in the study of wireless communication of nuclear power plants. The research shows that it is an NP problem to improve the coverage of wireless sensor networks, and the swarm intelligence algorithm can be used to optimize the coverage of nodes and get the approximate optimal solution. In this paper, based on the modeling of metal barrier channel and the unique advantages of teaching and learning algorithm in swarm intelligence algorithm, the network coverage is optimized and the redundancy of nodes is reduced.

3.1. Communication Coverage. In this paper, we build a twodimensional probability-aware wireless sensor network and calculate the coverage of the network. First, $N$ sensor nodes are randomly deployed in the $L * L$ monitoring area, and the wireless sensors are completely the same. For any point $P(x, y)$ in the monitoring area, $d_{1}\left(s_{i}, P\right)$ is the Euclidean distance between sensor nodes $s_{i}$ and $P$, namely, $d_{1}\left(s_{i}, P\right)=\sqrt{\left(x-x_{i}\right)^{2}+\left(y-y_{i}\right)^{2}}$. The relationship between the probability accuracy and distance of a single sensor is given according to the following equation:

$$
c_{s i}(P)= \begin{cases}1, & d_{1}\left(s_{i}, P\right) \leq r, \\ e^{-\rho \alpha^{\beta}}, & r<d_{1}\left(s_{i}, P\right) \leq R, \\ 0, & d_{1}\left(s_{i}, P\right)>R,\end{cases}
$$

where $\beta$ and $\rho$ are adjustable parameters of the perceptual probability model $[13,14]$. Considering the characteristic that the perceptual probability of nodes decreases with the increase of perceptual distance, take $\rho=0.1$ and $\beta=0.7$. $R$ is the sensing radius of each sensor, $r$ is the sensing threshold, $r<R$ is the radius of deterministic region, and $\alpha=d_{1}\left(s_{i}, P\right)-r$.

At the same time, the $L * L$ monitoring area is homogenized into $L * L$ grids. The target point covered by the network is the intersection point of grids, and its set is $m$. At this time, the coverage rate of the wireless sensor network is the average of the probabilities of all sensed points in the monitoring area:

$$
w=\frac{1}{M} \sum p_{R}
$$

where $M$ represents the number of all target points in the monitoring area and $p_{R}$ is the probability when the target point is sensed by multiple sensors [15]. 
The problem of network coverage is to study how to make every point in the monitoring area covered by wireless sensor networks. Therefore, the research content of coverage problem can be transformed into optimizing the position of nodes, so that nodes can move from redundant areas to coverage blind areas, at which time the coverage area becomes larger and the coverage rate is enhanced.

\subsection{Improving the Teaching and Learning Algorithm. As a} new swarm intelligence algorithm, teaching and learning algorithm has the characteristics of few parameters and strong convergence [16-18]. However, research shows that this algorithm, like other swarm intelligence algorithms, has the disadvantages of local falling into the optimal solution and weak global convergence. In order to increase the application value of the algorithm, the teaching and learning algorithm is improved to enhance the searching ability of the algorithm and reduce the situation that the algorithm falls into the local optimal solution. Therefore, an adaptive disturbance strategy is proposed for the teaching and learning algorithm.

Teaching and learning algorithm is a kind of swarm intelligence algorithm proposed by simulating class learning. The improvement of students' grades in class requires not only teachers' "teaching" but also students' "learning." However, in the original algorithm, it was not considered that each member would forget himself or study independently, which would lead to fluctuation of achievement, which might lead to the algorithm falling into local optimum. Considering adding disturbance, students will have changes in their own level caused by forgetting and selfstudy in the whole algorithm optimization stage, thus enriching the diversity of students. The equation of adding disturbance is shown as follows:

$$
X_{\text {new }}^{i}=a \times[0.5-\operatorname{rand}(0,1)]+X_{\text {old }}^{i},
$$

where $a$ is the value that controls the degree of forgetting and learning of students.

In the constructed wireless sensor network, the sensing nodes are optimized by using the teaching and learning algorithm, and the objective function of the algorithm is network coverage. The guiding idea of the algorithm optimization is to increase the network coverage through the reasonable layout of the node position when the number of sensor nodes is constant. The algorithm optimization steps are as follows:

(1) In optimization, the number of sensing nodes $N$ is determined first and then the parameters are initialized. The parameters to be optimized in the algorithm are the coordinates of each sensor, so the parameters to be optimized are an array of $2 * N$. The upper and lower limits of each parameter are also an array of $2 * N$, and then the appropriate population size and iteration times are selected.

(2) Initialize node coordinates.

(3) The coverage probability of each target point of the network is calculated, and the coverage of the whole network is obtained by using the overall coverage equation (17).

(4) Entering the "teaching" stage of algorithm and determining the optimal individual $X_{\text {teacher }}$, each student gets the value after learning through calculation, and then they compare according to the results before and after learning. If $F\left(X_{\text {new }}^{i}\right)>F\left(X_{\text {old }}^{i}\right)$, then $X_{\text {old }}^{i}=X_{\text {new }}^{i}$, keeping students with good adaptability.

(5) Entering the "learning" stage of the algorithm and getting a new solution $X_{\text {new }}^{i}$, if $F\left(X_{\text {new }}^{i}\right)>F\left(X_{\text {old }}^{i}\right)$, then $X_{\text {old }}^{i}=X_{\text {new }}^{i}$, keeping the students with good adaptability.

(6) Then, according to the student's self-disturbance strategy, the convergence of the algorithm is enhanced and the situation of falling into local optimum is reduced.

(7) Judge whether the end condition is met or not; otherwise, add 1 to the number of cycles and jump to the stage of Step 3 to continue the optimization; if yes, terminate the algorithm and output the best adaptive value.

\subsection{Communication Coverage in Barrier Areas Based on} Teaching and Learning. The area coverage problem has always been an important research direction of sensor networks. The traditional area coverage research is mostly limited to the ideal monitoring environment, while the research on the area coverage problem in complex environment is less. In the actual monitoring area, there are usually many different types of obstacles. If the sensing range of nodes intersects with obstacles, there will be many blind spots in the monitoring area, which will seriously affect the monitoring performance of sensor networks. Therefore, it is very important to design and optimize the deployment strategy of sensor nodes and dynamically adjust the working direction of sensors. In this paper, considering the influence of multiple obstacles in sensor networks, we propose a coverage algorithm based on teaching and learning algorithm to optimize the coverage of the monitoring area.

In order to be closer to the actual deployment environment of sensor nodes, it is necessary to consider the coverage of obstacles. In order to meet the general requirements, a number of random polygon obstacles are set in the monitoring environment. Six wireless sensor network nodes are predeployed in the monitoring area of $100 \mathrm{~m} \times 100 \mathrm{~m}$ as nodes connected with wireless gateway, and the positions of these six nodes are given. On this basis, a certain number of nodes are deployed to study how to maximize the coverage.

In this study, in order to meet the communication requirements in the nuclear reactor cabin environment, the coverage radius of sensor nodes is not the conventional coverage radius, but refers to the radius of node communication. Considering the complex deployment environment, the acceptance sensitivity in this study is $-35 \mathrm{~dB}$ to ensure sufficient received signal strength. The coverage area 
of each node changes with the environment. If there are obstacles nearby, the coverage area becomes smaller; otherwise, free space communication is used to give the communication coverage radius. The specific communication scope is as follows: where $P_{r}(d)$ is the path loss when the distance between transceiver equipment is $d$, and the unit is $\mathrm{db} ; G_{T}$ is the transmitter antenna gain; $G_{R}$ is the receiver antenna gain; $P_{r}\left(d_{0}\right)$ indicates the reference path loss at short distance $d_{0}$ (generally taken as $1 \mathrm{~m}$ ), which is obtained from actual test; $n$ is the path loss index, which indicates the rate at which the path loss increases with distance, and it depends on the surrounding environment and building type; $X_{\sigma}$ represents a normal random variable with standard deviation $\sigma$, and considering environmental factors, the value of $\sigma$ generally varies from 3.0 to $14.1 \mathrm{~dB}$. In equation (16), there are two parts: line-of-sight communication and non-line-of-sight communication. Line-of-sight communication is available when there are no obstacles, and the communication distance is greatly shortened when there are obstacles.

If additional sensor nodes $\left\{S_{1}, S_{2}, \ldots, S_{m}\right\}$ are randomly deployed on the basis of preset sensors in a two-dimensional closed plane $\psi$ with an area of $100 \mathrm{~m} \times 100 \mathrm{~m}$, the network coverage rate is

$$
C=\frac{\bigcup_{i=1}^{m} a_{i}}{A},
$$

where $C$ represents coverage, rate $A$ represents the whole area, $a_{i}$ represents the area covered by the $i$ th node, and $m$ is the number of nodes.

\section{Simulation}

4.1. MATLAB Simulation. Before the experiment, the model and algorithm parameters are determined first. Parameters of terrain model $l_{x}, l_{y}$ is 100 meters, respectively. In the process of communication, the transmitting and receiving gain of the transmitted packet length antenna is 1 and the communication frequency $f$ is $433 \mathrm{MHz}$. Each experiment was repeated 30 times. 30 times is a widely accepted value that can reach the statistical conclusion. The parameters of the teaching and learning algorithm here are set as population number 10 and iteration number 30 .

Based on the teaching and learning algorithm, the communication coverage optimization of obstacle areas is studied. First, six sensor nodes are preset in the network, which are fixed in position and connected to the corresponding wireless gateway, respectively, and then the communication coverage of the network is formed by introducing other nodes.

The coverage of $10,11,12,13$, and 14 nodes is studied through the calculation of teaching and learning algorithm. It can be seen from Table 1 in each case that the optimized network coverage rate is obviously improved; compared with randomization, the average coverage rate of the whole network increased by $8.21 \%$. Furthermore, it can be seen from Table 1 that the network coverage is not necessarily high in the randomized state, although there are many nodes, but with the optimization of the algorithm, the network coverage will definitely be improved with the increase of the number of nodes. This also shows that optimizing the coverage of wireless sensor networks through teaching and learning algorithms can steadily improve the overall coverage of the network.

The optimization of coverage can also be analyzed through the historical iteration curve of the algorithm. It can be seen from Figure 11 that no matter what kind of node situation, with the increase of iteration times, the overall coverage of the network has been significantly improved. Because of the randomness of the algorithm itself, the convergence degree of the algorithm is not consistent with the number of nodes, showing a certain randomness, but it has reached a certain convergence at the end of the iteration.

In addition to the comparison of coverage data and the analysis of coverage optimization by covering iterative curves, the node coverage graph can also be used for intuitive observation, as shown in Figures 12-16. Among them, the white area indicates the obstacle area, the green point indicates the covered point, and the red plus sign indicates the sensor node position. Obstacle areas are not completely inaccessible areas, which need to rely on the algorithm itself to avoid obstacles. It can be seen from the following comparison diagrams from 10 nodes to 15 nodes that the optimization effect of each pair of comparison diagrams after covering is obvious compared with that before covering. Moreover, longitudinal observation shows that, with the increase of the number of nodes, the optimized coverage rate is obviously better.

Finally, in order to verify the validity and reliability of the method studied in this paper, it is also compared and verified 
TABLE 1: Improvement of coverage optimization degree.

\begin{tabular}{|c|c|c|c|c|c|}
\hline Overlay node & 10 & 11 & 12 & 13 & 14 \\
\hline Not optimized & 0.8611 & 0.8921 & 0.9081 & 0.8868 & 0.9266 \\
\hline After the optimization & 0.9441 & 0.9514 & 0.9679 & 0.9825 & 0.9952 \\
\hline Lifting rate & $9.64 \%$ & $6.65 \%$ & $6.59 \%$ & $10.79 \%$ & $7.40 \%$ \\
\hline
\end{tabular}
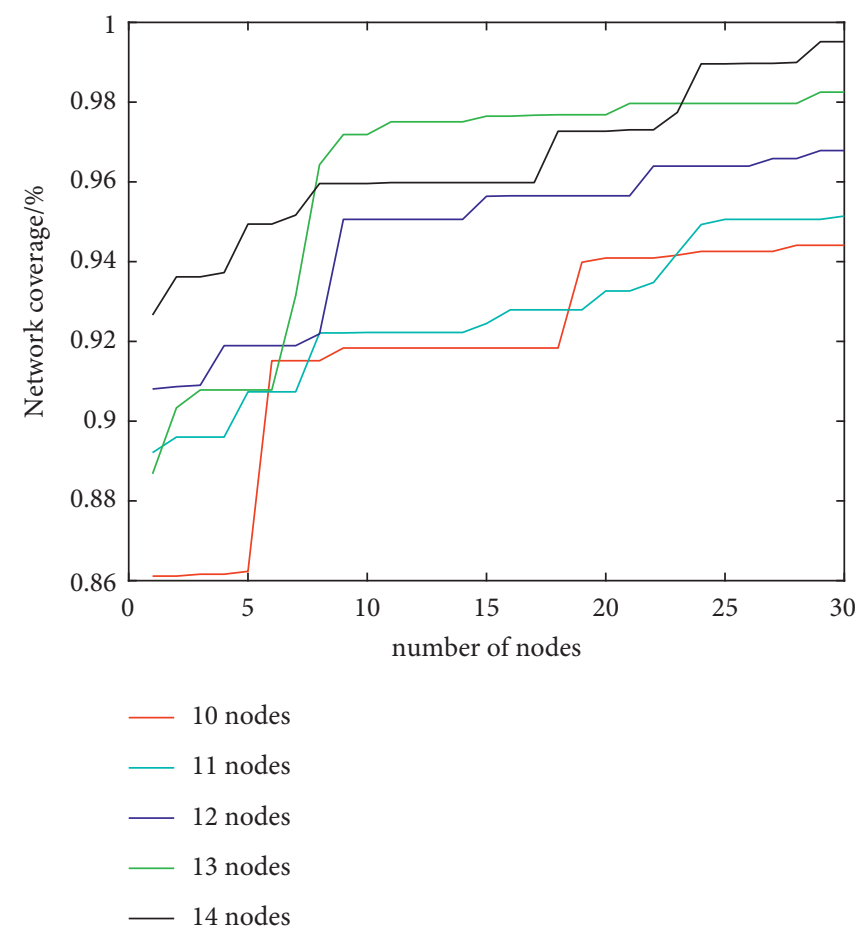

FIgURE 11: Comparison of coverage optimization with different node numbers.

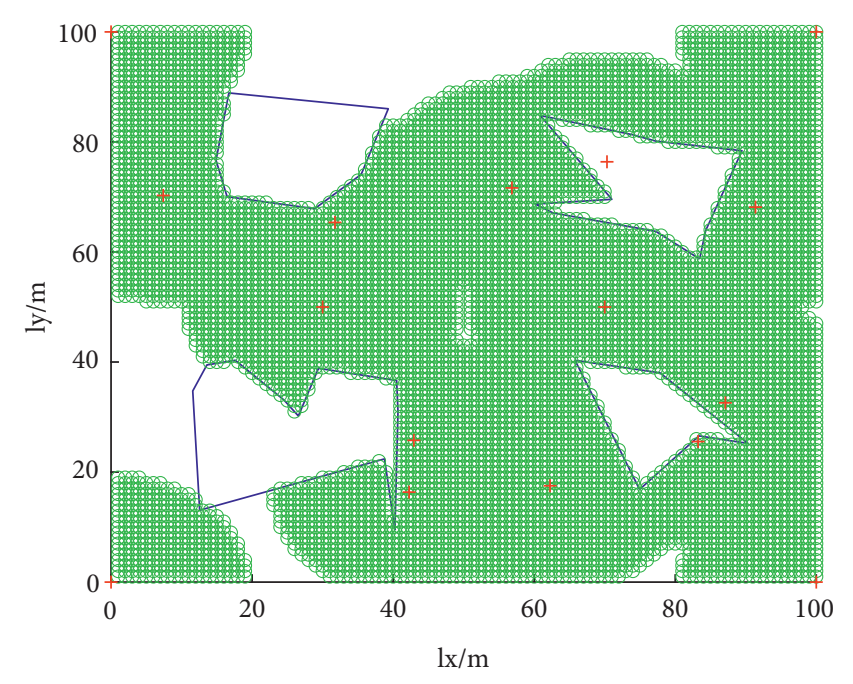

(a)

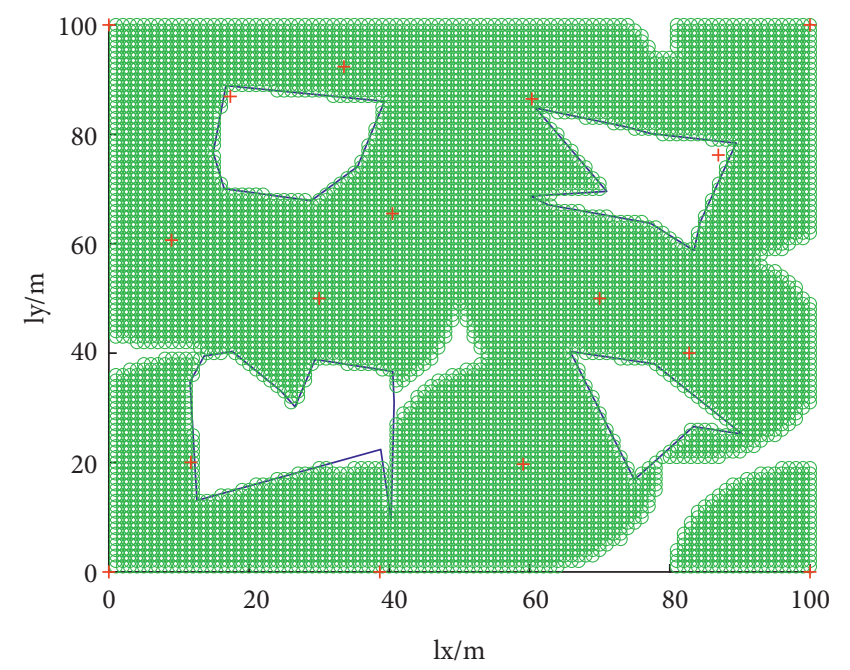

(b)

FIGURE 12: Network coverage of 10 nodes: (a) before optimization and (b) after optimization.

with the existing common channel modeling methods, ray method and time-domain finite difference method, and the comparison results are shown in Table 2.
From the coverage comparison results in Table 2, it is clear that the method in this paper has the highest coverage and is the most effective. 


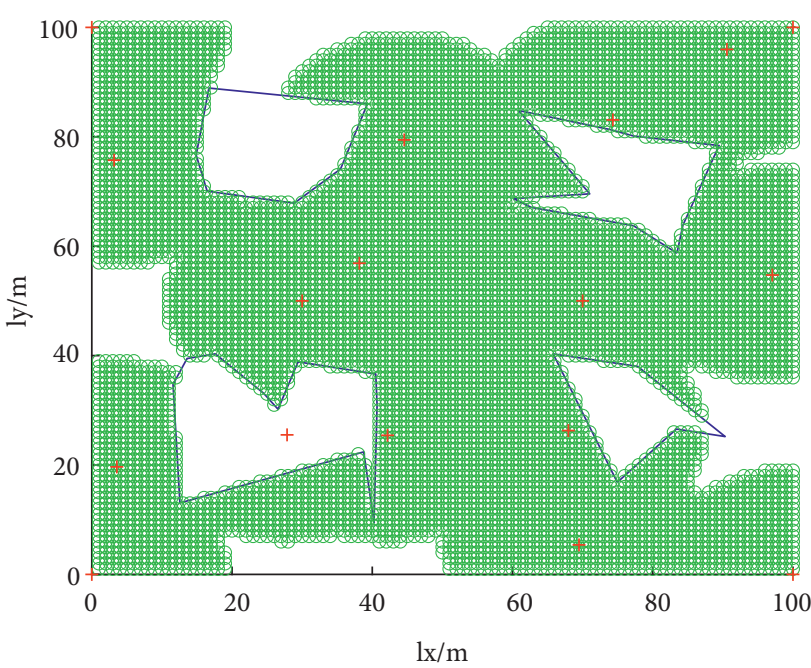

(a)

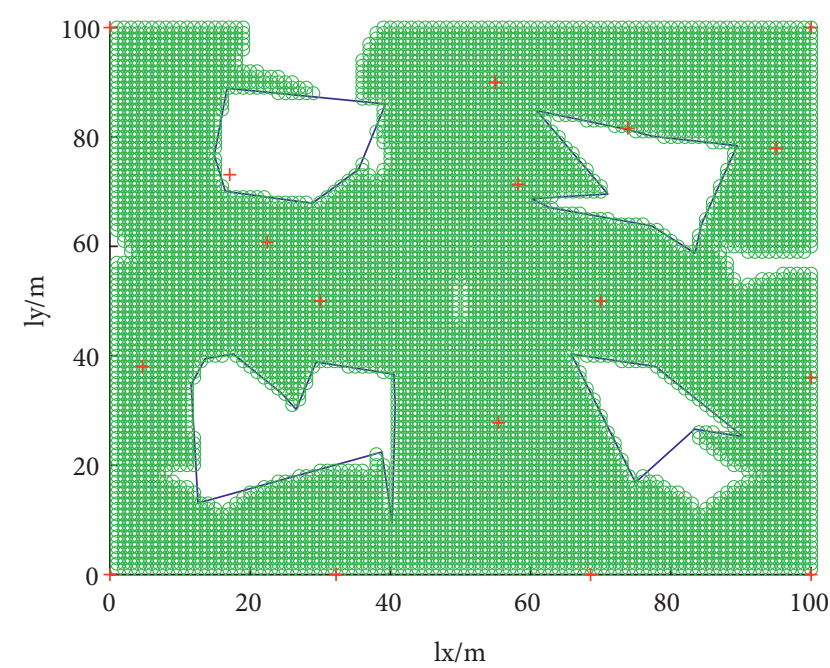

(b)

Figure 13: Network coverage of 11 nodes: (a) before optimization and (b) after optimization.

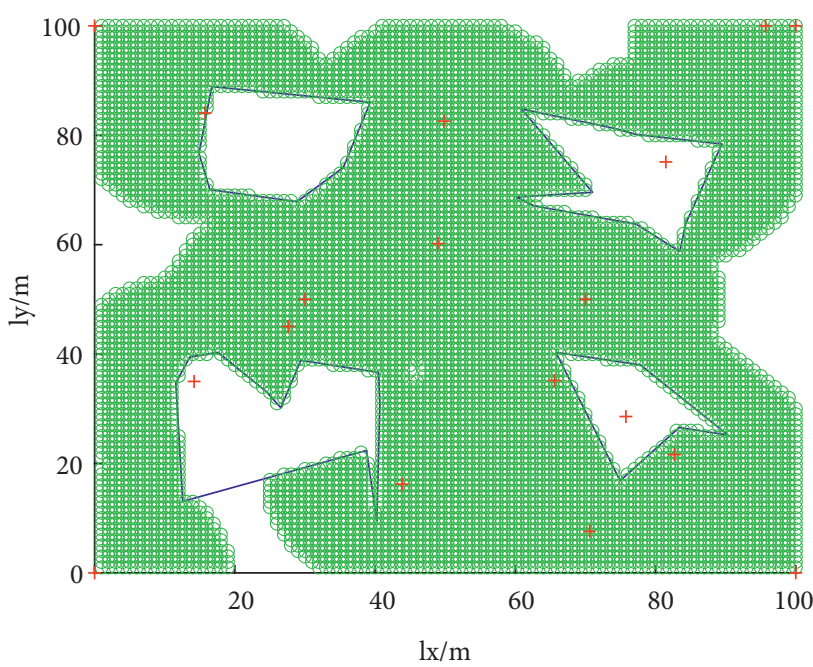

(a)

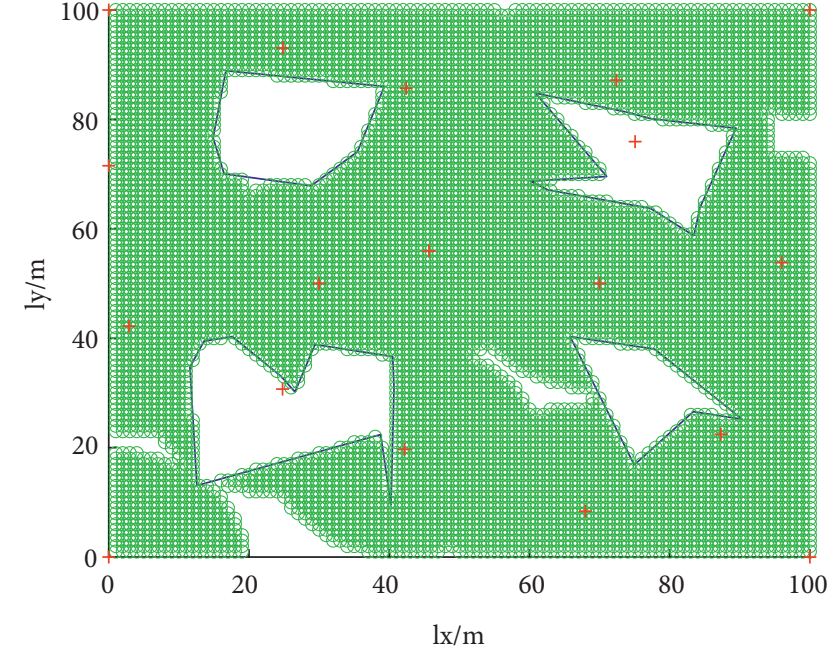

(b)

FIGURE 14: Network coverage of 12 nodes: (a) before optimization and (b) after optimization.

4.2. Bench Validation. To further verify the effectiveness of the method proposed in this paper, based on the method described in this paper, the main obstacles in the multimetal barrier in the bench, which is similar to the actual environment of the ship, are abstractly modeled, and the optimized arrangement of wireless sensors is implemented by the method in this paper, followed by the communication packet loss rate and coverage test to verify. As shown in Figure 17, eight wireless temperature sensors are arranged in an area of about $13 \mathrm{~m} \times 13 \mathrm{~m}$ in the bench, keeping the position of the wireless gateway at the receiver fixed at the position shown in G1 in the diagram of the second floor of the bench, and the communication packet loss rate of the wireless sensors at each position is analyzed by keeping the data transmission over 30 minutes. At the same time, by adding specific wireless temperature sensors (it should be noted that because it is a group intelligence algorithm to optimize the coverage, there are nodes arranged in obstacles in Section 4.1, i.e., in the white area, the actual arrangement in moving the nodes arranged in obstacles to the positions around the obstacles), the coverage of the whole area network is analyzed.

The wireless temperature sensor arranged in the first layer S7 position is shown in Figure 18, which shows that the metal blocking around it is very serious, and the data display of the upper computer $\left(18.96^{\circ} \mathrm{C}\right)$ is shown in Figure 19, 


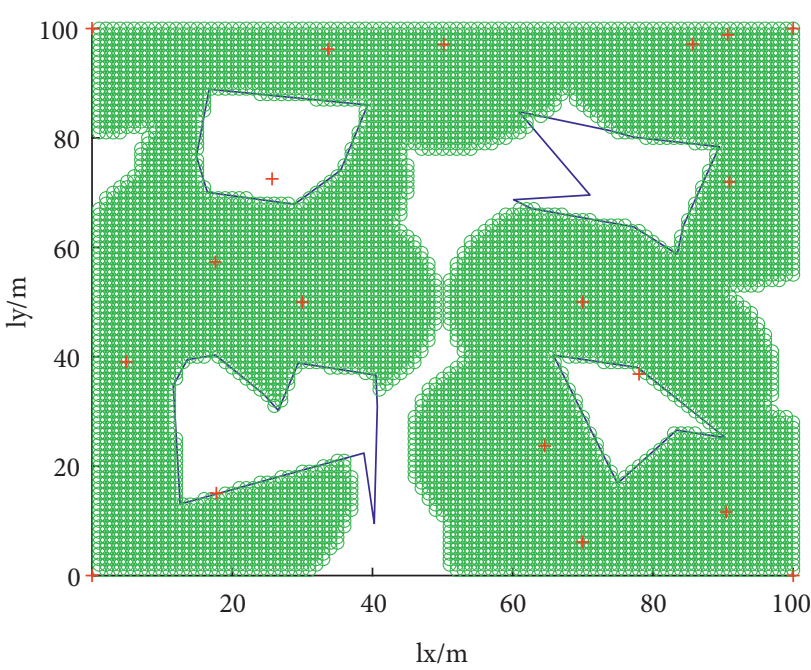

(a)

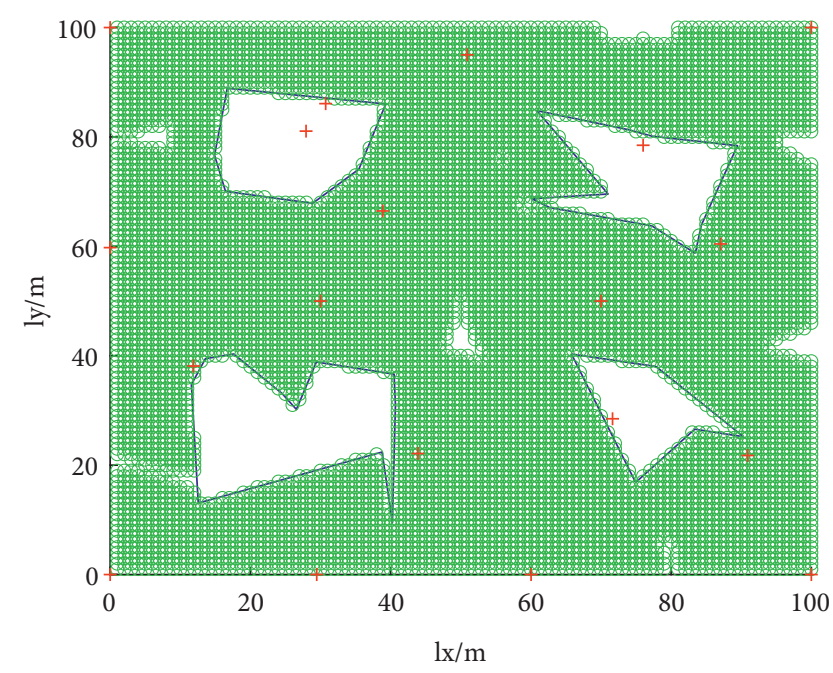

(b)

FigURE 15: Network coverage of 13 nodes: (a) before optimization and (b) after optimization.

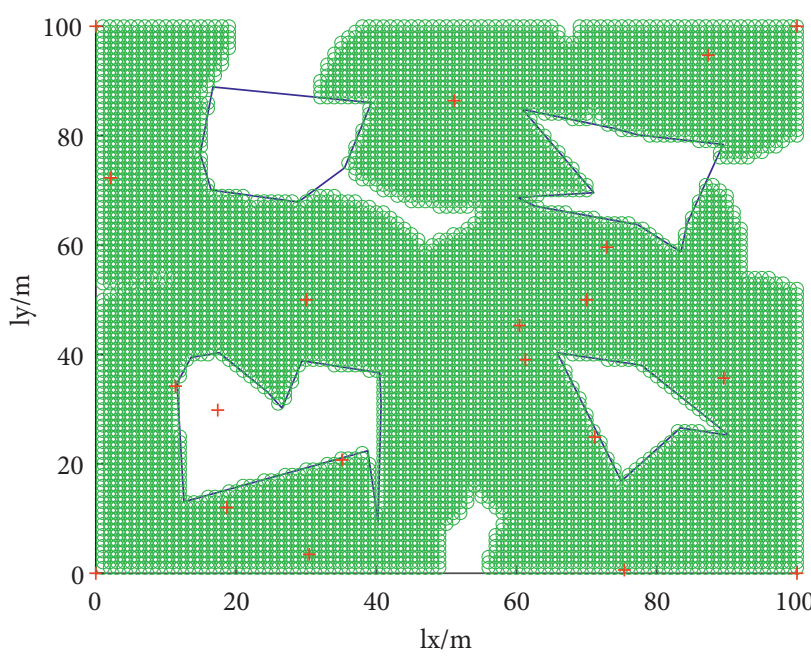

(a)

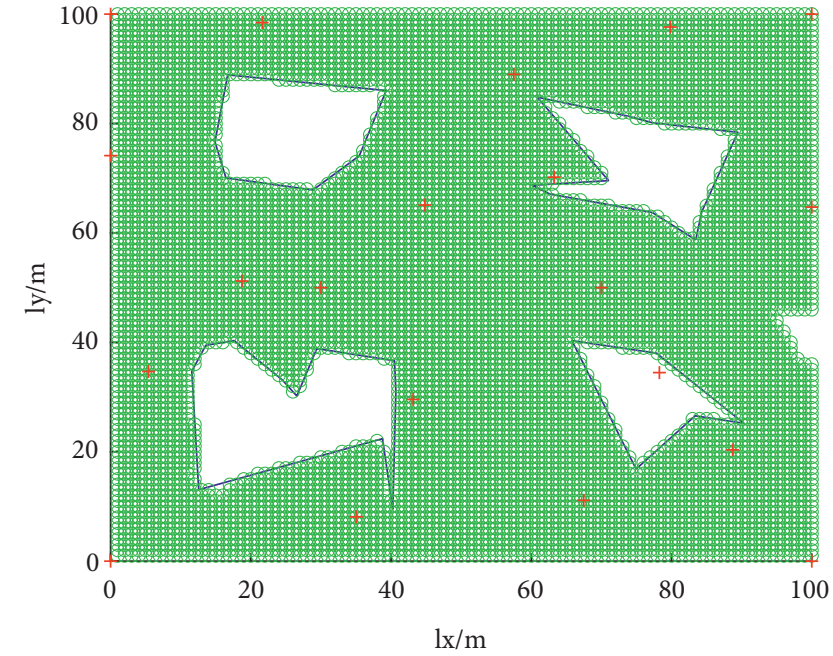

(b)

FIGURE 16: Network coverage of 14 nodes: (a) before optimization and (b) after optimization.

TABLE 2: Comparison of coverage rates of different algorithms with different number of nodes.

\begin{tabular}{|c|c|c|c|c|c|}
\hline Channel modeling methods & 10 & 11 & 12 & 13 & 14 \\
\hline Method of this article & 0.9441 & 0.9514 & 0.9679 & 0.9825 & 0.9952 \\
\hline Radio frequency method & 0.9161 & 0.9421 & 0.9589 & 0.9667 & 0.9792 \\
\hline Time-domain finite difference method & 0.9223 & 09433 & 0.9601 & 0.9808 & 0.9896 \\
\hline
\end{tabular}

which shows that the data can still be received normally in such a complicated environment.

The packet loss rate of the wireless temperature sensors at locations S1-S8 is shown in Figure 20. The packet loss rate of the wireless temperature sensor data at all locations is less than $0.5 \%$, and the S1-S8 data fluctuate around the average value $\left(19.20^{\circ} \mathrm{C}\right)$. It can be seen that the wireless temperature sensor can achieve the wireless temperature measurement 


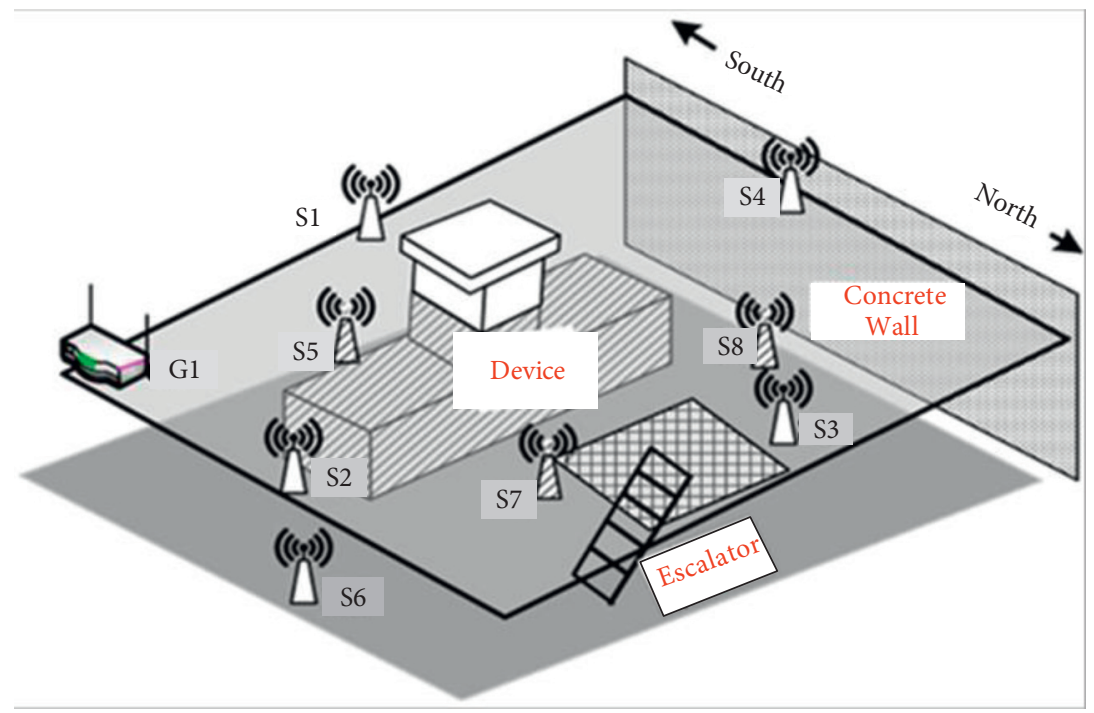

FIGURE 17: Simulation bench test of wireless sensor network engine room environment.

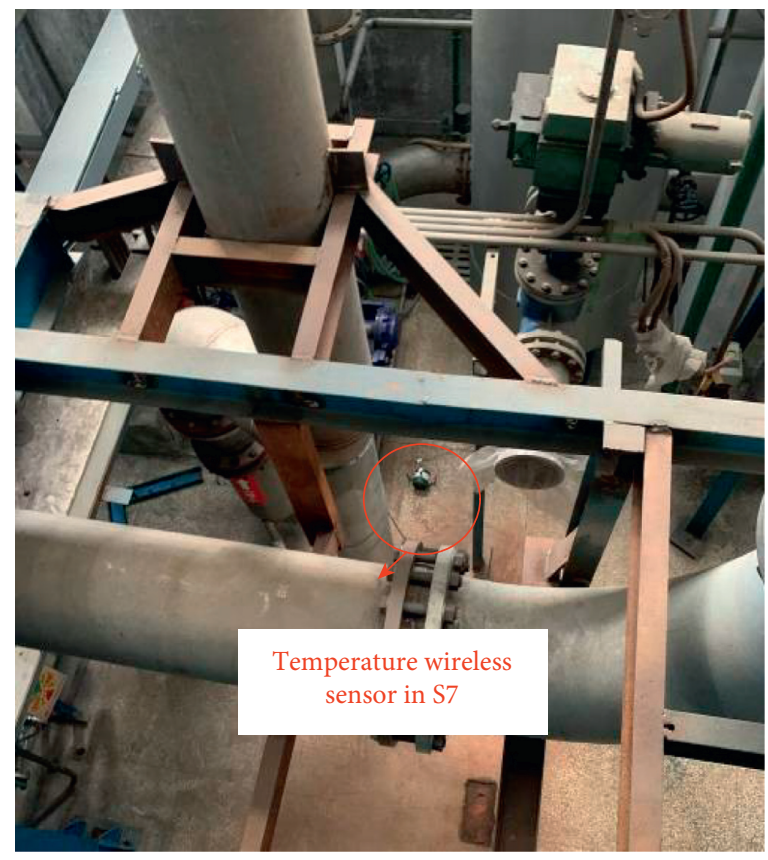

FIGURE 18: Display of the wireless temperature sensor at the S7 position on the first floor of the gantry.

function with a small packet loss rate intact after the optimized arrangement based on channel modeling in the multimetal barrier environment.

Based on the simulation results of Section 4.1, wireless temperature sensors were gradually added in specific areas to test the area coverage. The arrangement of one particular location is shown in Figure 21, where a wireless temperature sensor is arranged behind a tank (in operation at about $30^{\circ} \mathrm{C}$ ) and the linear communication path between it and the wireless gateway is completely obscured by the tank. Figure 22 shows the upper computer data $\left(25.32^{\circ} \mathrm{C}\right)$, which show that the communication can still be established under such severe conditions. 


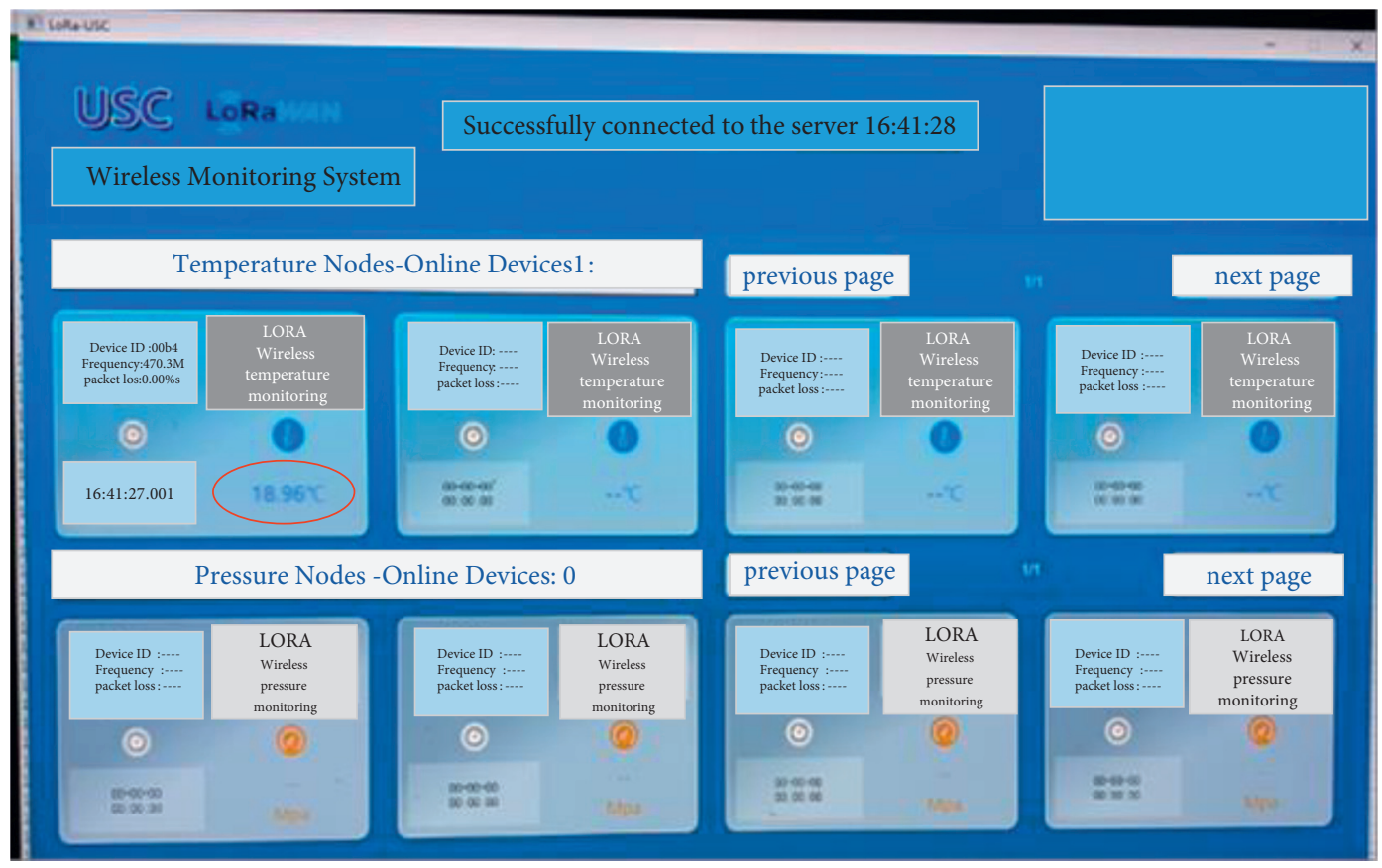

Figure 19: Wireless temperature sensor value display at S7 on the first level of the bench.
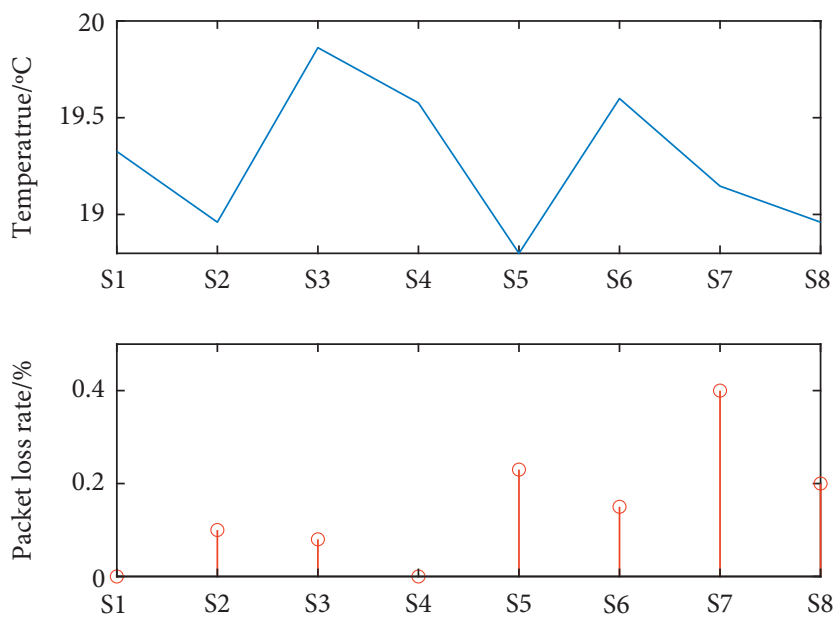

Figure 20: Packet loss rate and temperature of wireless temperature sensors at S1-S8 positions.

After increasing the number of location-specific wireless temperature sensors to 7 , which means the total number of sensors increases to 15 , the area coverage reaches more than $99 \%$, as shown in Table 3. From Table 3, it can be seen that the coverage of the bench test is always lower than that of the simulated case with the same number of wireless sensor node arrangements, which is in line with the actual situation because the actual bench has many other small obstacles in addition to the main obstacle being abstractly modeled, which will hinder the communication to some extent. Also, the wireless sensor arrangement of the actual bench is not exactly the same as the simulated result, as the simulated nodes that fall in the obstacle in the simulation are moved next to the obstacle. 

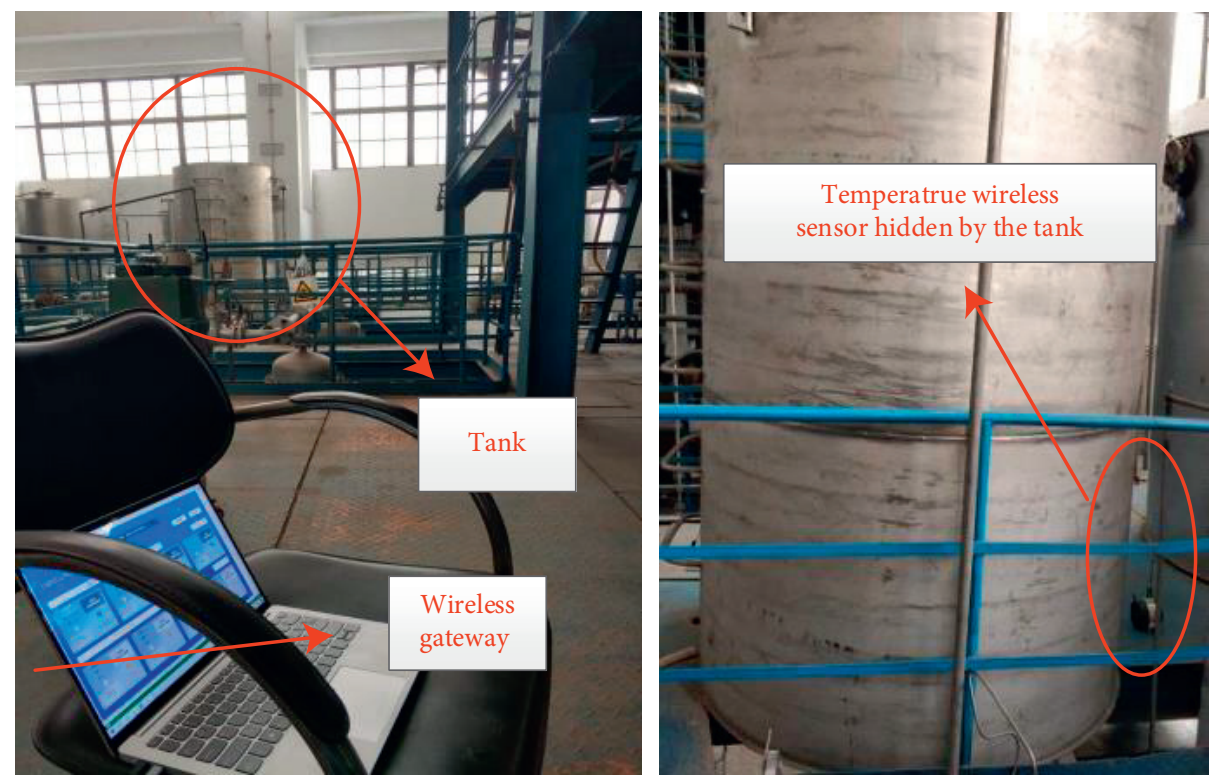

Figure 21: Wireless temperature sensor arrangement at a special location on the second floor of the platform.

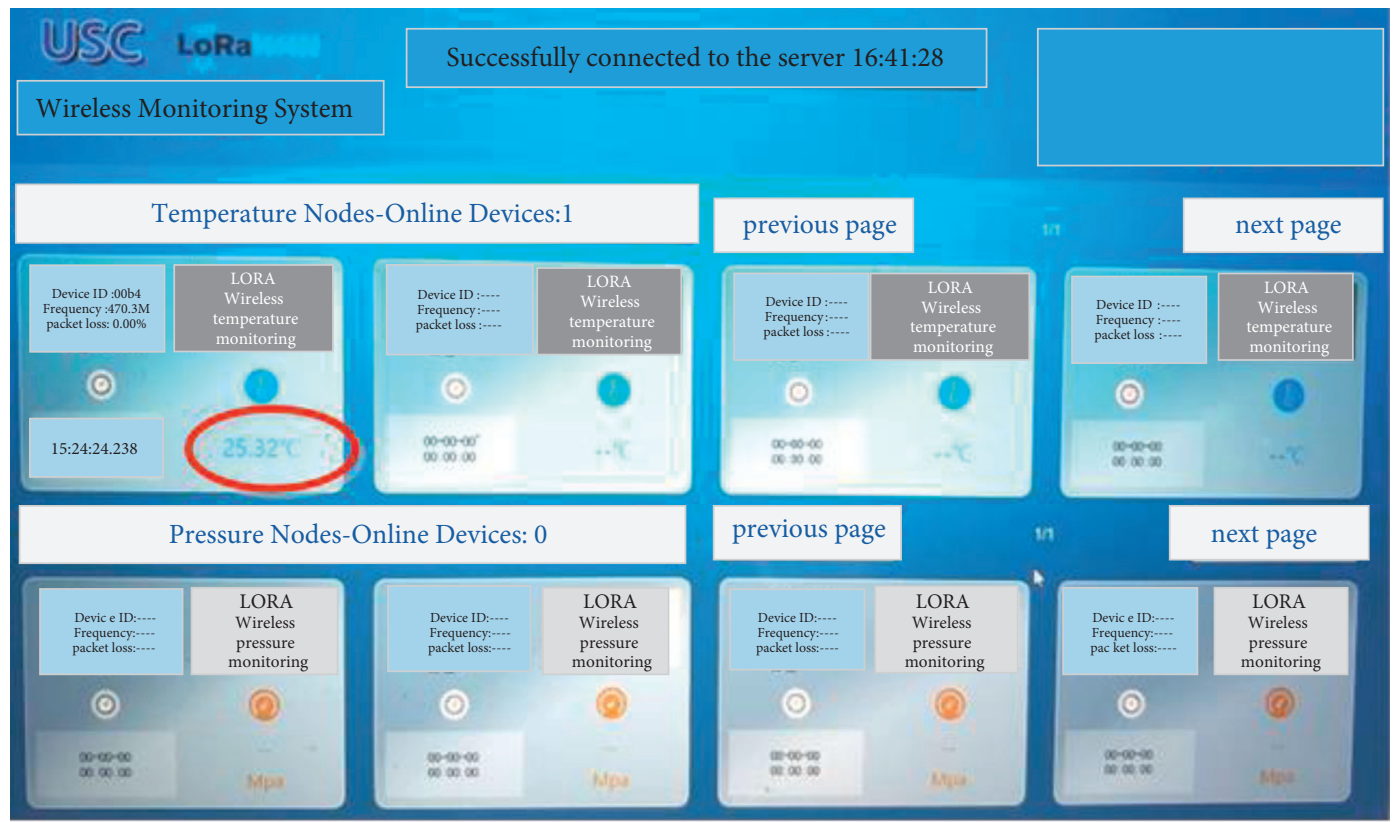

Figure 22: Display of wireless temperature sensor values at specific locations on the second level of the table.

TABLE 3: Comparison of simulation and actual bench test coverage.

\begin{tabular}{lcccccc}
\hline Test situation & 10 & 11 & 12 & 13 & 14 & 15 \\
\hline MATLAB simulation & 0.9341 & 0.9467 & 0.9601 & 0.9752 & 0.9882 & 0.9972 \\
Bench testing & 0.9123 & 09334 & 0.9571 & 0.9648 & 0.9796 & 0.9901 \\
\hline
\end{tabular}

\section{Conclusion}

By considering the interference factors such as transmit power, transmit position, and metal, this paper models the barrier area channel and concludes that higher transmit power can improve the received signal quality, smoother metal surface can reduce the loss of wireless channel transmission, and appropriate transmit angle and transmit position can also reduce the multipath propagation of wireless channel, so that the quality of channel reception is 
better. At the same time, on the basis of channel modeling, by adding disturbance factors to improve the teaching and learning swarm intelligence algorithm, the network coverage of the obstacle area is optimized. The simulation results show that the proposed algorithm has good effect. Compared with the initial state, the optimized network coverage is significantly improved, and the overall average network coverage is improved by more than $8 \%$ compared with the randomization. At the same time, by gradually increasing the number of nodes, we can see that, in the case of four obstacles, 14 nodes can cover more than $99 \%$ of the whole area. Finally, this paper also conducts the verification test of communication packet loss rate and coverage rate on the test bench to prove the effectiveness of the method in this paper.

\section{Data Availability}

No data were used to support this study.

\section{Conflicts of Interest}

The authors declare that they have no conflicts of interest.

\section{References}

[1] Q. Li, Research on Large-Scale Multi-Antenna Channel Measurement and Channel Fading Characteristics, pp. 1-10, Beijing Jiaotong University, Beijing, 2017.

[2] Z. Deng, Q. Wu, X. Lv, S. Zhu, X. Xu, and X. Wang, “Application analysis of wireless sensor networks in nuclear power plant," in Proceedings of the the Fourth International Symposium on Software Reliability, Industrial Safety, Cyber Security and Physical Protection of Nuclear Power Plant (ISNPP), pp. 135-148, Guiyang, China, 2020.

[3] Z. Qin, Wireless Channel Modeling Based on 3D Ray Tracing, p. 20, Southeast University, Jiangsu, 2018.

[4] X. Zhou, "Modeling of wireless transmission channel in confined space," Journal of Nanyang Institute of Technology, vol. 7, pp. 27-30, 2015.

[5] H. Q. Huang, W. Liu, P. R. Wu, and M. H. Xia, "Status and prospects of machine learning in wireless channel modeling," Mobile Communications, vol. 45, no. 04, pp. 95-104, 2021.

[6] J. He, J. Li, and A. Yang, "Research on high-speed channel modeling based on machine learning methods," Computer Engineering and Science, vol. 43, no. 06, pp. 984-988, 2021.

[7] L. Liu, J. Zhang, Y. Fan, L. Yu, and J. Zhang, "A review on the application of machine learning in channel modeling," Journal of Communication, vol. 42, no. 02, pp. 134-153, 2021.

[8] W. Wang, Principles and Systems of Mobile Communication, Beijing University of Posts and Telecommunications, Beijing, 2005.

[9] J. Wei, N. Liu, Y. Hei, J. Tang, X. Xing, and J. Wei, "Experiments on multipath effect in wireless communication," Experimental Technology and Management, vol. 37, no. 10, pp. 77-79, 2020.

[10] M. Li, Research on Channel State Information Acquisition Technology of Large-Scale MIMO System, 12 pages, Chongqing University of Posts and Telecommunications, Chongqing, 2019.

[11] W. Xu, Research on Channel Modeling and Multipath Clustering Algorithm Based on Scattering Clusters, pp. 12-14, Tianjin University, Tianjin, 2012.
[12] J. Wang, "Design of SNR estimator in aviation wireless communication system," Aerospace Electronics Technology, vol. 45, pp. 6-9, 2014.

[13] Y. M. Ren and Y. G. Niu, “A directed sensor fence coverage enhancement algorithm under a perceptual probability model," Small Microcomputer Systems, vol. 39, no. 01, pp. 122-127, 2018.

[14] L. P. Jiang, L. M. Wang, S. M. Xiong, and Y. Z. Zhan, "Sensing probability-based k-recovering algorithm for wireless sensor networks," Computer Application Research, vol. 26, no. 09, pp. 3484-3486, 2019.

[15] Q. Qi, Research on Coverage Algorithm of Wireless Sensor Networks Based on Probabilistic Perception Model, Huazhong University of Science and Technology, Huazhong, 2012.

[16] Y. He, B. Qian, and R. Hu, "Hybrid discrete teaching and learning algorithm for complex parallel machine scheduling problem," Acta Automatica Sinica, vol. 46, no. 4, pp. 805-819, 2020.

[17] L. Ma, P. Li, and L. Yun, "Interval optimal operation of wind power generation system based on teaching and learning algorithm," Electric Drive Automation, vol. 41, no. 5, pp. 19-23, 2019.

[18] S. Wang, H. Zhang, and J. Sun, "Test data generation of pairwise combination of improved teaching and learning algorithms," Journal of Xi'an University of Posts and Telecommunications, vol. 25, no. 2, pp. 41-45, 2020. 